\title{
ÉMISSION D'ÉLECTRONS AUGER PAR BOMBARDEMENT IONIQUE MÉCANISME ET POSSIBILITÉ D'APPLICATION A L'ANALYSE DES SURFACES $(*)$
}

\author{
P. VIARIS DE LESEGNO et J.-F. HENNEQUIN
}

C. N. R. S., Laboratoire P. M. T. M., Université Paris-Nord

Avenue Jean-Baptiste Clément, 93430 Villetaneuse, France

(Reçu le 30 décembre 1976, accepté le 24 février 1977)

\begin{abstract}
Résumé. - L'émission d'électrons Auger au cours du bombardement ionique de cibles solides est régie par le même mécanisme que l'émission des cibles gazeuses, c'est-à-dire par la possibilité de transitions électroniques aux croisements des orbitales moléculaires formées au cours d'une collision suffisamment violente. Une meilleure connaissance du phénomène serait nécessaire pour qu'on puisse l'appliquer à l'analyse quantitative des surfaces solides.
\end{abstract}

\begin{abstract}
The mechanism of Auger electron emission by ion bombardment of solid targets is similar to that of gaseous targets. It depends on the possibility of electronic transitions at crossings of molecular orbitals formed during sufficiently violent collisions. A better knowledge of this mechanism would be necessary in order to apply the phenomenon to quantitative analysis of solid surfaces.
\end{abstract}

1. Introduction. - Lors de l'étude des collisions entre les molécules d'un gaz raréfié et des ions rapides (à des énergies supérieures au kiloélectron-volt), on observe l'émission de photons $\mathrm{X}$ ou d'électrons Auger caractéristiques de l'un ou l'autre des partenaires $[1,2,3]$. En effet, durant une collision, il se produit une promotion des orbitales moléculaires, qui peut conduire à des transitions électroniques et donc, après séparation, à la création d'un trou dans un niveau électronique interne de l'une des particules. La désexcitation ultérieure s'effectue avec l'émission, soit d'un photon $\mathbf{X}$, soit d'un électron Auger. Pour les atomes de bas numéro atomique, l'effet Auger est le processus prépondérant; il se traduit par un pic à une énergie bien déterminée, caractéristique de l'atome émetteur, dans le spectıe d'énergie des électrons secondaires.

Le mécanisme de promotion des orbitales moléculaires $[4,5]$ est maintenant bien connu et rend compte de façon satisfaisante des expériences de collisions en phase gazeuse. Si la cible est un solide, le processus de collision entre le projectile et un atome de la cible reste fondamentalement inchangé, tant que l'énergie de la collision est suffisante pour que la présence des autres atomes de la cible n'influe pas directement sur les deux atomes en collision.

(*) Communication présentée au Congrès National de Physique des Plasmas, Paris, 8 décembre 1976.
Cependant, du fait de la très forte densité du solide, le projectile peut subir plusieurs collisions successives à énergie élevée avec des atomes de la cible et mettre ainsi en mouvement rapide un grand nombre d'entre eux. Un atome rapide de la cible peut dès lors lui aussi entrer en collision violente avec un autre atome de la cible et produire un trou dans un niveau électronique interne d'un de ces atomes après une collision symétrique, dont l'effet se superposera à celui des collisions asymétriques entre le projectile et les atomes de la cible (où le trou peut se former selon les cas dans l'un ou l'autre des atomes partenaires).

Deux autres conséquences sont liées à l'utilisation de cibles solides. La région de la cible qui contribue à l'émission Auger est limitée à une profondeur de l'ordre du libre parcours moyen des électrons étudiés dans le solide (soit quelques couches atomiques); il s'agit donc d'une émission pratiquement superficielle. D'autre part, le spectre d'énergie des électrons Auger, c'est-à-dire le profil du pic Auger, est modifié par l'intervention d'électrons de la bande de conduction $[6,7]$.

On peut mettre en évidence la coexistence des deux types de collisions dans les expériences où une collision asymétrique ne peut conduire à la création d'un trou électronique que dans un niveau interne du seul projectile. Cette situation se rencontre lorsque le numéro atomique du projectile est légèrement inférieur à celui de la cible $[3,8,9]$, par exemple lors du bombardement de cibles de magnésium ou d'alu- 
minium par des ions sodium $\mathrm{Na}^{+}$[10] ou néon $\mathrm{Ne}^{+}$[11]. On observe alors (Fig. 1) sur un même spectre le pic Auger caractéristique du projectile (sodium), résultant des seules collisions asymétriques, et le pic Auger du métal cible (magnésium ou aluminium), résultant des seules collisions symétriques.

2. Mécanisme de formation du trou interne. $-\mathrm{La}$ formation du trou électronique interne s'interprète de manière satisfaisante dans le cadre de la théorie des orbitales moléculaires [4]. Au cours de la collision, il se forme une quasi-molécule dont on peut prévoir par le calcul les niveaux électroniques. On trace ainsi un diagramme de corrélation qui représente l'évolution des niveaux électroniques avec la distance internucléaire, depuis une distance infinie à laquelle correspondent les niveaux non perturbés des deux atomes séparés, jusqu'à la distance pratiquement nulle à laquelle correspondent les niveaux non perturbés de l'atome unifié, c'est-à-dire l'atome dont le numéro atomique est la somme des numéros atomiques des deux atomes partenaires en collision. Un diagramme de corrélation grossier peut être tracé en tenant compte uniquement des règles de symétrie des diverses orbitales $[5,12]$; il permet de prévoir dans la plupart des cas le niveau électronique où pourra se former un trou. Seul le calcul explicite à l'ordinateur de l'évolution des niveaux permet en outre de connaître la distance internucléaire en deçà de laquelle la formation du trou devient possible.

Un trou électronique peut en effet être créé dans une orbitale initialement pleine du fait de l'interaction de cette orbitale avec une autre orbitale incomplètement occupée, soit par couplage radial (s'il y a croisement d'orbitales), soit par couplage rotationnel (si les orbitales sont énergétiquement proches). Au cours de l'interaction, un électron transite d'une orbitale à une autre, pouvant ainsi laisser subsister un trou électronique interne dans l'un des atomes après séparation.

Si les atomes en collision sont de numéros atomiques voisins, les niveaux électroniques de l'atome de plus bas numéro atomique sont les plus externes (parce que l'attraction coulombienne du noyau est moins forte); ils forment donc des liaisons antiliantes qui permettent, après croisement ou du moins interaction avec des orbitales incomplètement occupées, la création du trou sur l'un des niveaux électroniques internes de l'atome de plus bas numéro atomique. Cette conclusion, bien vérifiée expérimentalement aux énergies pas trop élevées [8,9], justifie l'interprétation que nous avons donnée du spectre Auger de la figure 1.

Il est bien évident que, dans le cas d'atomes identiques (collisions symétriques), le trou électronique peut se former sur l'un ou l'autre des partenaires. Pour les éléments légers $(10 \leqslant Z \leqslant 18)$, les principaux pics Auger observés correspondent à des transitions faisant intervenir un trou dans le niveau $2 p$ de l'atome

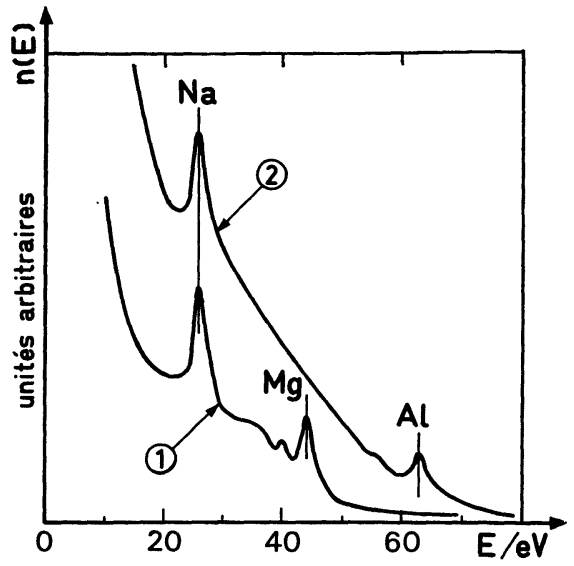

Fig. 1. - Spectres Auger de cibles de magnésium (courbe 1) et d'aluminium (courbe $2 ; \times 2$ ) bombardées par des ions $\mathrm{Na}^{+}$ d'une énergie de $12 \mathrm{keV}$.

[Auger spectra of magnesium (curve 1) and aluminium (curve 2 ; $\times 2$ ) targets bombarded with $12 \mathrm{keV} \mathrm{Na}{ }^{+}$ions.]

émetteur $[6,7,13,14,15,16]$. Le trou se forme facilement dans le niveau $2 p$ parce que l'orbitale moléculaire $4 \mathrm{f} \sigma$, corrélée au niveau $2 \mathrm{p}$ de l'atome de plus bas numéro atomique (ou de l'un des deux atomes s'ils sont identiques) subit une promotion importante sur une très petite variation de la distance internucléaire à une distance critique $r_{\mathrm{c}}$ relativement grande, à laquelle correspond une énergie seuil qui dépend des partenaires en collision et qui varie d'une fraction de kiloélectron-volt à quelques $\mathrm{keV}[3,15]$. La transition ne peut pas se produire tant que l'énergie de collision est notablement inférieure à l'énergie seuil, et aucun pic Auger n'est observé dans ce cas. Lorsque l'énergie de collision est supérieure à l'énergie seuil, la transition peut avoir lieu selon que la distance de plus proche approche est ou non inférieure à $r_{\mathrm{c}}$ : on observe donc une croissance très rapide de l'intensité du pic Auger avec l'énergie de collision, jusqu'à atteindre un palier quand l'énergie de collision est très supérieure à l'énergie seuil. L'émission du néon semble présenter un comportement particulier, avec une lente diminution de l'intensité du pic Auger au-dessus d'une énergie de bombardement de $1,2 \mathrm{keV}$ environ [11].

3. Résultats expérimentaux. - 3.1 ÉMISSION AUGER DU PROJECTILE. - L'émission d'électrons Auger caractéristiques $\mathrm{du}$ projectile lors du bombardement d'un solide est susceptible d'une interprétation quantitative, car les collisions qui peuvent conduire effectivement à l'observation d'électrons Auger ont lieu dans des conditions assez bien définies :

- l'épaisseur efficace de la cible est limitée à quelques couches atomiques (ordre de grandeur du libre parcours moyen des électrons Auger observés) ; elle ne semble pas dépendre du métal cible [15] et est très inférieure à la profondeur moyenne de pénétration des ions incidents ; 
- l'énergie de collision dans les premières couches atomiques est donc pratiquement égale à celle des ions incidents, un ralentissement notable ne pouvant résulter que d'une collision avec un atome de la cible.

Seules interviennent dans l'émission Auger du projectile les collisions binaires asymétriques des ions incidents et des atomes de la cible. Une collision dans les premières couches atomiques d'un ion incident sur un atome de même nature précédemment implanté semble constituer un événement peu probable. En effet, bien que la section efficace de formation d'un trou interne soit maximale pour une telle collision symétrique, l'intensité du signal Auger demeure proportionnelle à l'intensité du bombardement et aucun effet de dose (quantité d'ions incidents implantés) n'a pu être mis en évidence $[11,15]$. En outre, l'émission Auger du projectile ne s'observe que lorsque le numéro atomique du projectile est inférieur à celui du métal cible.

L'émission Auger de l'argon (énergie des électrons Auger voisine de $200 \mathrm{eV}$ ) a ainsi pu être étudiée en bombardant des métaux de numéro atomique supérieur à celui de l'argon $(\mathrm{K}, \mathrm{Ca}, \mathrm{Sc}, \mathrm{Ti}, \mathrm{V}, \mathrm{Cr})$ avec des ions $\mathrm{A}^{+}$au voisinage de l'énergie du seuil [15]. Les résultats expérimentaux permettent d'obtenir la section efficace de collision qui peut aussi être calculée numériquement. On détermine ainsi la distance critique $r_{\mathrm{c}}$, la largeur de la zone de transition $\left({ }^{\mathbf{1}}\right)$ et l'épaisseur efficace de la cible (pour laquelle a été trouvée la valeur constante : $12 \AA$ pour tous les métaux de transition étudiés).

Avec les métaux légers (magnésium, aluminium, etc...), l'émission Auger de l'argon ne s'observe pas, car l'émission Auger du projectile nécessite des ions incidents plus légers que les atom $: \mathrm{s}$ de la cible. La figure 1 présente les résultats que nous avons obtenus avec des ions $\mathrm{Na}^{+}[10]$; ils n'ont malheureusement pas pu faire l'objet d'une exploitation aussi complète que ceux obtenus avec l'argon, le spectromètre magnétique utilisé étant mal adapté à des mesures quantitatives d'intensité au-dessous de $20 \mathrm{eV}$. Pour la même raison, nous avons dû renoncer à une étude quantitative de l'émission du néon. Par contre, Ferrante et Pepper [11], disposant d'un analyseur cylindrique, ont récemment obtenu, lors du bombardement de cibles de magnésium et d'aluminium avec des ions $\mathrm{Ne}^{+}$au voisinage du seuil d'émission Auger du néon, des résultats similaires à ceux obtenus avec des ions $\mathrm{A}^{+}$sur les métaux de transition.

Les valeurs de la distance critique $r_{\mathrm{c}}$ que l'on peut

(1) La distance critique $r_{\mathrm{c}}$ correspond en réalité à la distance internucléaire où la probabilité de transition d'un électron d'un niveau interne à un niveau externe devient égale à la moitié de la probabilité maximale. La largeur de la zone de transition est l'intervalle de distance sur laquelle la probabilité de transition passe en gros du quart à la moitié de la valeur maximale. ainsi déduire des mesures d'intensité d'émission Auger au voisinage du seuil d'émission sont en bon accord avec celles obtenues dans les expériences en phase gazeuse, par mesure de la perte d'énergie des ions incidents [9] ou par mesure de l'intensité de l'émission $\mathrm{X}$ caractéristique [3]. Il est intéressant aussi de comparer $r_{\mathrm{c}}$ à la somme $R$ des rayons électroniques (plus précisément les rayons de densité électronique maximale) des couches $2 p$ des deux atomes en collision [17] (dans le cas du néon, un doute subsiste sur la couche à considérer, mais le rayon de la couche $2 \mathrm{~s}: 0,37 \AA$ ne diffère guère de celui de la couche $2 p: 0,32 \AA$ ). On constate ainsi (Fig. 2) que la transition s'effectue à une distance internucléaire où le recouvrement des couches est important, de l'ordre de $0,1 \AA$.

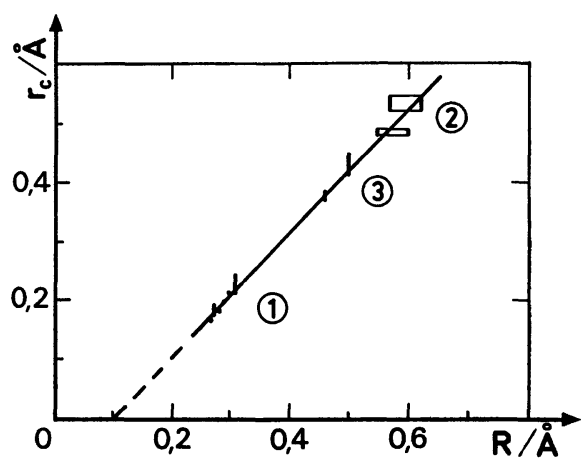

FIG. 2. - Distance critique de transition $r_{\mathrm{c}}$ en fonction de la somme $R$ des rayons des couches $2 p$ des atomes en collision. Série de points $1: \mathrm{A}^{+} \rightarrow \mathrm{A}[3,9], \mathrm{K}, \mathrm{Ca}, \mathrm{Sc}, \mathrm{Ti}, \mathrm{V}[15]$; série 2 : $\mathrm{Ne}^{+} \rightarrow \mathrm{Mg}, \mathrm{Al}[11]$; série $3: \mathrm{Mg} \rightarrow \mathrm{Mg}$ et $\mathrm{Al} \rightarrow \mathrm{Al}$ par bombardement $\mathrm{Ne}^{+}, \mathrm{A}^{+}, \mathrm{Kr}^{+}$et $\mathrm{Xe}^{+}$[19].

[Critical transition distance $r_{\mathrm{c}}$ versus $R$, the sum of the $2 \mathrm{p}$-shell radii of the colliding atoms. Series $1: \mathrm{Ar}^{+} \rightarrow \operatorname{Ar}(3,9), \mathrm{K}, \mathrm{Ca}, \mathrm{Sc}$, Ti, V [15] ; series $2: \mathrm{Ne}^{+} \rightarrow \mathrm{Mg}$, Al [11] ; series $3: \mathrm{Mg} \rightarrow \mathbf{M g}$ and $\mathrm{Al} \rightarrow \mathrm{Al}$ by bombardment of the corresponding metals with $\mathrm{Ne}^{+}, \mathrm{Ar}^{+}, \mathrm{Kr}^{+}$and $\mathrm{Xe}^{+}[19]$.]

La détermination quantitative de la composition de la surface d'un échantillon cible comportant plusieurs espèces atomiques peut s'envisager, en choisissant convenablement le projectile selon les éléments étudiés et en opérant au voisinage des seuils d'émission. La procédure consisterait à identifier tout d'abord les éléments présents, par exemple par leur émission Auger caractéristique (voir $\S 3.2$ ) pour les numéros atomiques inférieurs à 29 (le cuivre semble bien constituer une limite de détection [18]), par leur émission $\mathrm{X}$ au-delà. Tenu compte de la section efficace de collision d'un élément à l'énergie de bombardement utilisée, chaque élément intervient dans l'émission Auger du projectile proportionnellement à sa concentration dans les premières couches atomiques de la cible. Si donc on connaît la variation avec l'énergie des sections efficaces d'émission Auger du projectile avec les différents partenaires possibles, on peut en principe déduire la composition de la surface de la cible de la variation, avec l'énergie de 
bombardement, de l'intensité du pic Auger du projectile. Cette mesure ne devrait faire intervenir que la quantité globale de l'élément dans la couche analysée, et non la constitution chimique de la cible (composé ou mélange, alliage en solution homogène ou comportant des précipités, etc...).

3.2 Émission Auger de la cible. - Les deux types de collisions, symétriques et asymétriques, sont susceptibles d'intervenir simultanément dans l'émission Auger des atomes de la cible. Même lorsque les conditions de bombardement sont telles que seules les collisions symétriques peuvent conduire à l'émission caractéristique, l'exploitation des résultats est plus délicate, car les conditions de collision ne sont pas bien déterminées.

L'énergie de collision est mal définie dans les collisions symétriques, car une collision asymétrique antérieure est nécessaire pour mettre en mouvement un atome de la cible, dont l'énergie est donc comprise entre l'énergie de déplacement dans le solide et l'énergie maximale transmissible dans la collision asymétrique (c'est-à-dire lors d'un choc de plein fouet). Tout au plus peut-on admettre que l'énergie seuil de l'émission Auger caractéristique coïncide avec cette énergie maximale transmissible. C'est ainsi que sont déterminées les valeurs de $r_{\mathrm{c}}$ présentées à la figure 2 qui correspondent aux collisions symétriques $\mathrm{Mg} \rightarrow \mathrm{Mg}$ et $\mathrm{Al} \rightarrow \mathrm{Al}$, à partir des résultats de Ferrante et Pepper [19] et en accord avec les nôtres.

En outre, dans le cas où la cible contient plusieurs espèces atomiques, divers types de collisions peuvent éventuellement conduire à la formation d'un trou électronique interne dans un atome cible donné, et donc contribuer ensemble à l'émission Auger de l'élément considéré. L'émission Auger caractéristique d'un élément de la cible permet sans difficultés l'identification de cet élément, mais l'intensité de l'émission ne dépend pas simplement de la concentration dans la cible et ne semble pas pouvoir conduire à une analyse quantitative commode. On remarquera que, si les collisions symétriques sont suffisamment efficaces, l'intensité du pic Auger caractéristique d'un élément devrait varier notablement, pour une même quantité globale, selon que l'élément considéré est dilué dans un alliage ou concentré dans des agglomérats (à l'intérieur desquels les collisions symétriques sont favorisées).

Une méthode d'étude des profils de diffusion ou d'implantation qui tend à se développer actuellement, consiste à décaper progressivement par bombardement ionique l'échantillon étudié, tout en analysant la surface en spectrométrie Auger classique par bombardement électronique. Dans le cas des métaux légers dont l'émission Auger sous bombardement ionique est particulièrement intense, le signal Auger risque d'être fortement perturbé par la présence du bombardement de décapage $[16,20]$. L'analyse peut encore s'effectuer, soit en pulsant le faisceau ionique de décapage et en procédant aux analyses Auger quand le faisceau est interrompu, soit, ce qui est expérimentalement plus facile, en introduisant une modulation d'intensité dans le faisceau électronique d'analyse et en détectant le signal Auger par démodulation synchrone. Ces complications de mesure ne paraissent pas nécessaires avec les métaux de transition dans les conditions usuelles d'opération.

4. Conclusion. - Le mécanisme de promotion des orbitales moléculaires, bien établi pour les collisions en phase gazeuse, s'applique encore convenablement aux collisions violentes à l'intérieur d'un solide et permet d'interpréter les émissions d'électrons Auger et de rayons $\mathrm{X}$ lors du bombardement ionique de cibles solides. Une meilleure connaissance des sections efficaces de formation de trous électroniques internes permettrait d'envisager l'application de l'émission Auger par bombardement ionique à l'analyse quantitative des surfaces, mais une étude approfondie des divers types de collisions impliquées dans l'émission serait auparavant nécessaire.

\section{Bibliographie}

[1] Rudd, M. E., Jorgensen, Jr, T. et Wolz, D. J., Phys. Rev. 151 (1966) 28.

[2] CaCaK, R. K. et Jorgensen Jr, T., Phys. Rev. A 2 (1970) 1322.

[3] Saris, F. W., Physica 52 (1971) 290.

[4] Fano, U. et Lichten, W., Phys. Rev. Lett. 14 (1965) 627.

[5] Lichten, W., Phys. Rev. 164 (1967) 131.

[6] Hennequin, J.-F., J. Physique 29 (1968) 1053.

[7] Viel, L., BenaZeth, C. et BenaZeth, N., Surface Sci. 54 (1976) 635.

[8] Fastrup, B. et Hermann, G., Phys. Rev. Lett. 23 (1969) 157.

[9] Fastrup, B., Hermann, G. et Smith, K. J., Phys. Rev. A 3 (1971) 1591.

[10] Viaris De Lesegno, P., Rivais, G. et Hennequin, J.-F., Phys. Lett. 49A (1974) 265.

[11] Ferrante, J. et Pepper, S. V., Surface Sci. 57 (1976) 420.

[12] Barat, M. et Lichten, W., Phys. Rev. A 6 (1972) 211.
[13] Viel, L., Colombié, N., Fagot, B. et Fert, Ch., C. R. Hebd. Séan. Acad. Sci. B 271 (1970) 239.

[14] Hennequin, J.-F. et Viaris de Lesegno, P., C. $R$. Hebd. Séan. Acad. Sci. B 273 (1971) 1259 ; Surface Sci. 42 (1974) 50.

[15] Viaris de Lesegno, P. et Hennequin, J.-F., J. Physique 35 (1974) 759.

[16] Grant, J. T., Hooker, M. P., Springer, R. W. et HaAs, T. W., J. Vac. Sci. Techn. 12 (1975) 481.

[17] Slater, J. C., Quantum Theory of Atomic Structure (MacGraw-Hill Book Comp., New York), I (1960) 210.

[18] Viel, L., Fagot, B. et Colombié, N., C. R. Hebd. Séan. Acad. Sci. B 272 (1971) 623.

[19] Ferrante, J. et Pepper, S. V., Surface Sci. 58 (1976) 613.

[20] Strausser, Y. E., Luscher, P. E. et Johannesson, J. S., VIth Symposium on Surface Physics : the Solid-Vacuum Interface, Eindhoven, 1976. 